\title{
An unusual adverse event of azathioprine: Cutaneous small vessel vasculitis
}

\author{
Shunichiro Hanai $₫$, Kei Kobayashi $[$, Daiki Nakagomi $₫$ \\ Third Department of Internal Medicine, University of Yamanashi, Chuo-shi, Yamanashi, Japan
}

In this article, we present a Japanese female case of cutaneous small vessel vasculitis (CSVV) after administration of oral azathioprine (AZA).

A 56-year-old Japanese woman with limited cutaneous systemic sclerosis developed a dry cough three years after the diagnosis and was referred to our hospital for further assessment. Skin thickening of her fingers and forearms was observed, but neither erythema nor purpura was present. Serum C-reactive protein (CRP) was $0.1 \mathrm{mg} / \mathrm{dL}$, and anti-nuclear antibody was positive (1:1,280 with nucleolar and discrete speckled pattern); anti-centromere, anti-Scl-70, anti-RNA polymerase III, and myeloperoxidaseand proteinase 3-antineutrophil cytoplasmic antibodies were negative. Computed tomography revealed a mild interstitial pneumonia. She received oral cyclophosphamide $50 \mathrm{mg}$ per day for the treatment of skin and pulmonary involvement, as she refused intravenous cyclophosphamide therapy. Since oral cyclophosphamide for one year had no effect on the pulmonary involvement, it was discontinued, and the patient was switched to daily oral AZA $50 \mathrm{mg}$ the next day. Ten days after the administration of AZA, she developed palpable and painful erythema on both extremities (Figure 1a, b). Serum CRP level was elevated to $13.8 \mathrm{mg} / \mathrm{dL}$, while complete blood count, serum transaminase, and creatinine levels remained unchanged. Skin biopsy was performed, and AZA was discontinued. The biopsy revealed leukocytoclastic vasculitis in the dermis, consistent with CSVV (Figure 1c-f). One week after cessation of AZA, her erythema almost completely resolved, and serum CRP level decreased to $0.83 \mathrm{mg} / \mathrm{dL}$. Due to the immediate improvement after cessation of AZA, she was diagnosed with CSVV associated with AZA, and oral cyclosporine was administrated. She had no recurrence of erythema and developed no signs of systemic vasculitis for five years. Subsequent genotyping of nucleoside diphosphate-linked moiety X-type motif 15 (NUDT15) codon 139 showed an Arg/Arg genotype.

Although various AZA-associated cutaneous adverse events have been reported so far, only a few reports of CSVV have been published. Appelman and Wintzen ${ }^{1}$ described a woman with Crohn's disease who developed pruritus and CSVV due to AZA. Intriguingly, this patient was using AZA for two years, but vasculitis occurred after the daily dose of AZA was doubled. Beckett et al. ${ }^{2}$ also described a man with immune thrombocytopenia presenting with fever and CSVV nine days after AZA administration. The typical onset of CSVV after exposure to a trigger

Received: October 04, 2020 Accepted: December 11, 2020 Published online: January 14, 2021

Correspondence: Shunichiro Hanai, MD. Third Department of Internal Medicine, University of Yamanashi, Chuo-shi, 409-3898 Yamanashi, Japan. Tel: +81-55-273-9602 e-mail: shanai@yamanashi.ac.jp 

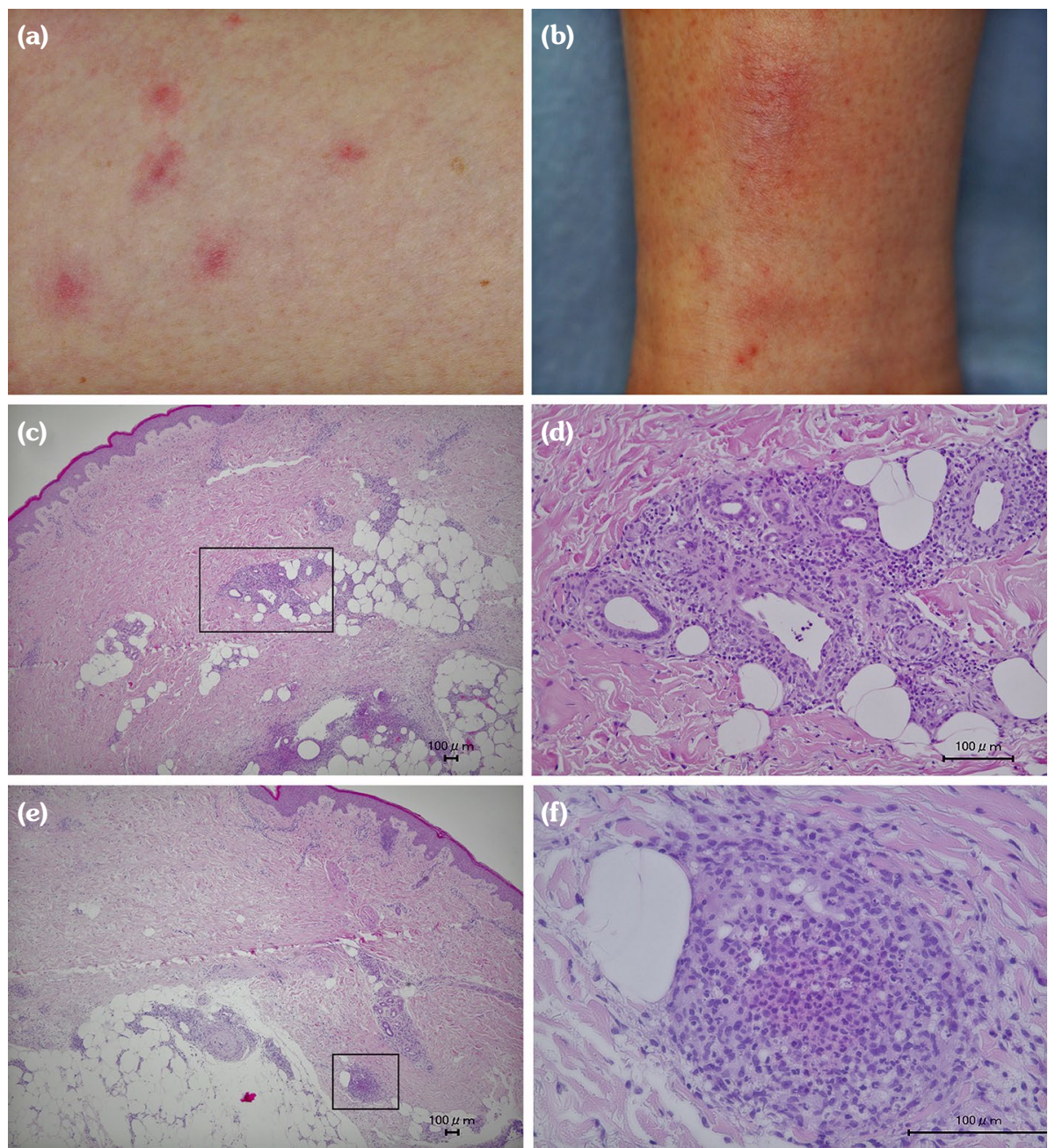

Figure 1. (a, b) Palpable erythema on lower extremities. (c-f). Skin biopsy showed leukocytoclastic vasculitis. Small vessels and perivascular tissue in the dermis were infiltrated with polymorphonuclear leukocytes and nuclear debris. There was no evidence of eosinophil infiltration. The vascular lumen was nearly obliterated (d-f).

such as a drug is seven to 10 days. ${ }^{3}$ The time from AZA administration to onset in our case was consistent with previous reports. However, unlike Appelman and Wintzen's ${ }^{1}$ report, the dose of AZA was low. Although she did not approve drug challenge tests to delineate the nature of the AZA adverse event, she had a total score of 8 on the Naranjo scale, estimating the probability of adverse drug reactions, indicating a probable adverse reaction to AZA. ${ }^{4}$

Adverse events associated with AZA can be divided into two categories: dose-dependent toxic effects and dose-independent hypersensitivity reactions. ${ }^{5}$ The former type includes leukocytopenia, and a variant in NUDT15 codon 139 predicts severe leukopenia rather than thiopurine S-methyltransferase deficiency, particularly in Japanese individuals. ${ }^{6}$ Adverse events of AZA associated with genotypes of codon 139 are shown in Table $1 .^{6}$ Our patient had an Arg/Arg genotype, which carries a low risk for leukopenia frequently found in Japanese. Triggering of the adverse event following low-dose AZA and genotyping of NUDT15 codon 139 suggested that the CSVV in our patient occurred via an allergic or non-allergic hypersensitivity reaction. In conclusion, clinicians should be aware of CSVV as an unusual adverse event following administration of AZA, an immunosuppressive agent used to treat vasculitis syndrome. 


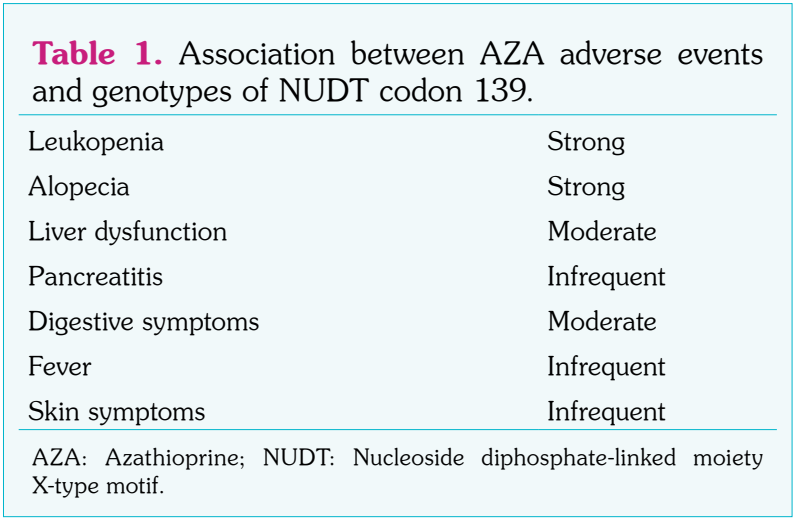

\section{Declaration of conflicting interests}

The authors declared no conflicts of interest with respect to the authorship and/or publication of this article.

\section{Funding}

The authors received no financial support for the research and/or authorship of this article.

\section{REFERENCES}

1. Appelman M, Wintzen M. Pruritus and leucocytoclastic vasculitis due to azathioprine. Dermatology 2005;210:366.

2. Beckett CG, Hill P, Hine KR. Leucocytoclastic vasculitis in a patient with azathioprine hypersensitivity. Postgrad Med J 1996;72:437-8.
3. Carlson JA, Cavaliere LF, Grant-Kels JM. Cutaneous vasculitis: diagnosis and management. Clin Dermatol 2006;24:414-29.

4. Naranjo CA, Busto U, Sellers EM, Sandor P, Ruiz I, Roberts EA, et al. A method for estimating the probability of adverse drug reactions. Clin Pharmacol Ther 1981;30:239-45.

5. Da Rosa GP, Marques S, Coelho F, Pereira E, Ferreira E, Rodrigues-Pereira $P$, et al. Azathioprine-induced interstitial nephritis in an anti-neutrophil cytoplasmic antibody (ANCA) myeloperoxidase (MPO) vasculitis patient. Eur J Rheumatol 2018;5:135-8.

6. Kakuta Y, Kawai Y, Okamoto D, Takagawa T, Ikeya $\mathrm{K}$, Sakuraba H, et al. NUDT15 codon 139 is the best pharmacogenetic marker for predicting thiopurineinduced severe adverse events in Japanese patients with inflammatory bowel disease: a multicenter study. J Gastroenterol 2018;53:1065-78. 